\title{
Erratum
}

\section{Measurement of Atomic Fractions in Multi-Phased Materials of Limited Mass via an Empirical Approach to EXAFS Modeling $\left(^{*}\right)$}

\author{
V.G. Harris $\left({ }^{1}\right)$, S.A. Oliver $\left({ }^{2}\right)$, J.D. Ayers $\left({ }^{1}\right)$ and B.N. Das $\left({ }^{1}\right)$ \\ ( ${ }^{1}$ U.S. Naval Research Laboratory, Washington, D.C. 20375, U.S.A. \\ $\left({ }^{2}\right)$ Center for Electromagnetics Research, Northeastern University, Boston, MA 02115, U.S A. \\ PACS.61.10.Ht - X-ray absorption spectroscopy· EXAFS, NEXAFS, XANES, etc. \\ PACS.61.43.Bn - Structural modeling: serial-addition models, computer simulation \\ PACS.64.70 Kb - Solid-solid transitions \\ PACS.68.35 Rh - Phase transitions and desorption kinetics; evaporation and condensation
}

\begin{abstract}
A least-square fitting analysis of EXAFS data collected from partially-crystallized $\mathrm{Fe}_{80} \mathrm{~B}_{20}$ thin films $(t=15 \mathrm{~nm})$, using data collected from pure phase standards of the crystallization products, was found effective in determining the relative atomic fraction of each crystalline phase present This fitting scheme provides a means for the quantitative treatment of crystalllization and precipitation kinetics in thin films and multilayered structures
\end{abstract}

A long standing limitation of extended X-ray absorption fine structure (EXAFS) as a tool for quantitative materials science has been its inability to measure the relative fraction of phases in multiphased materials. This is a prerequisite, for example, in the study of crystallization and precipitation kinetics. Although there exist techniques which are able to perform these tasks on bulk materials, e.g. X-ray diffraction and digital scanning calorimetry, recent trends toward the design and fabrication of low dimensional devices has made the study of thin films, which cannot be readily measured by these techniques because of their small masses, of particular importance. Of the popular local probes, EXAFS is largely insensitive to small masses [1], for example, the signal-to-noise ratio of the EXAFS collected in total electron yield mode does not deteriorate appreciably for thin films until the sampled mass approaches $\approx 10^{-9}$ grams. In an attempt to illustrate the usefulness of EXAFS in performing quantitative materials science we have applied EXAFS to study the crystallization of a model transition metal - metalloid amorphous system in Fe-B. A least-square fitting analysis of the EXAFS data collected from partially-crystallized Fe-B thin films, using data collected from pure phase standards of the crystallization products, was found effective in determining the relative atomic fraction of each crystalline phase present.

The samples used in this study are from a single $15 \mathrm{~nm}$ film ( $50 \mathrm{~mm} \times 50 \mathrm{~mm}$ area) which was ion beam sputter-deposited from a pressed-powder target having the stoichiometry of $\mathrm{Fe}_{80} \mathrm{~B}_{20}$. Individual pieces $(7 \mathrm{~mm} \times 7 \mathrm{~mm}$ ) were encapsulated in evacuated glass ampoules and annealed at temperatures ranging from $473 \mathrm{~K}$ to $823 \mathrm{~K}$ for a period of 60 minutes. The $\mathrm{X}$-ray absorption

$\left.{ }^{*}\right)$ This paper was published in the proceedings of the $9^{\text {th }}$ International conference on X-Ray Absorption Fine Structure, J. Phys IV France 7 (1997) C3-1153, with a wrong title. 
spectra, encompassing the Fe $\mathrm{K}$ edge, were collected using the total-electron-yield collection scheme $[2,3]$ on the X23B beamline at the National Synchrotron Light Source operating between $110 \mathrm{~mA}-230 \mathrm{~mA}$. The X23B beamline employs a double-crystal, fixed-exit, Si (111) monochromator with a collimating mirror for harmonic rejection. For the samples studied here the escape depth is greater than the film thickness hence the total volume of the sample exposed to the incident X-ray beam is measured. The EXAFS reduction analysis followed established procedures [4], which included the normalization of the extended fine structure to the absorption edge step height and energy, removal of a non-oscillatory blackground curvature, conversion to photoelectron wave vector $(k)$ space, and Fourier transformation to radial $(r)$ coordinates. Data were collected and similarly analyzed for an Fe foil, and tetragonal (t) $-\mathrm{Fe}_{2} \mathrm{~B}$ and $t-\mathrm{Fe}_{3} \mathrm{~B}$ powder samples which were used here as standards of the crystallization products The composition, $\mathrm{Fe}_{80} \mathrm{~B}_{20}$, has been found in bulk ribbons to crystallize near $710 \mathrm{~K}$ with the eutectic crystallization of $\alpha$ - $\mathrm{Fe}$ and $\mathrm{t}-\mathrm{Fe}_{3} \mathrm{~B}$. At higher temperatures the metastable $\mathrm{t}-\mathrm{Fe}_{3} \mathrm{~B}$ phase decomposes to $\alpha-\mathrm{Fe}$ and $\mathrm{t}-\mathrm{Fe}_{2} \mathrm{~B}$ phases [5-7].

In order to measure the atomic fraction of the different phases present in the annealed samples, the phase and amplitude of the EXAFS oscillations are $/$ fit in $k$-space using a linear combination of data sets collected from the pure phase standards. Unlike the more traditional approach to the quantitative fitting which typically includes fitting a limited range of Fourierfiltered $r$-space (typically the near neighbor environment) using single-scattering simulated EXAFS data, we fit an $r$-space range of $0-8 \AA$.

Figure 1 is a plot of the extended fine structure after normalization and conversion to $k$-space for an as-deposited $\mathrm{Fe}_{80} \mathrm{~B}_{20}$ film sample, and samples annealed at $473 \mathrm{~K}$ and $523 \mathrm{~K}$. In this plot one sees that the data for the as-grown sample appears qualitatively as a single frequency whose amplitude decreases with increasing wave vector. This is consistent with an amorphouslike structure where only the radial distances of the near-neighboring atoms are well-defined, hence a single frequency corresponding to these atoms appears in the EXAFS data. The data corresponding with the sample annealed at $473 \mathrm{~K}$ are similarly dominated by a single frequency, suggesting that this sample also has an amorphous-like structure.|Reduction in the amplitude of the oscillations over the low- $k$ region indicates changes in the local chemistry around the absorber which are likely due to changes in local atom configurations resulting from structural relaxation. In contrast, the sample annealed at $523 \mathrm{~K}$ illustrates a pronounced splitting near $5 \AA^{-1}$. This splitting is indicative of higher-order neighbors ordering around the absorbing atoms and is the first direct evidence that some medium- or long-range ordering has occurred in these samples.

In order to determine the relative fraction of the crystalline phases in the annealed samples, an $r$-space range of $0-8 \AA$ for each data set was Fourier-filtered to $k$-space and fit using a linear combination of similarly analyzed EXAFS data collected from the pure phase reference standards. Figure $1 \mathrm{~b}$ is a plot depicting the experimental, Fourier-filtered EXAFS data for the sample annealed at $573 \mathrm{~K}$ with the best fit obtained using a linear combination of $\alpha-\mathrm{Fe}, \mathrm{t}-\mathrm{Fe}_{3} \mathrm{~B}$ and amorphous $\mathrm{Fe}_{80} \mathrm{~B}_{20}$. The fit in Figure $1 \mathrm{~b}$ consists of $27.7 \pm 10 \%$ amorphous component, $40 \pm 3 \% \alpha-\mathrm{Fe}$, and $32.3 \pm 10 \% \mathrm{t}-\mathrm{Fe}_{3} \mathrm{~B}$. The only adjustable parameters in this fitting analysis were the relative amounts of each phase, and the radial distance and Debye-Waller term for the amorphous component. The parameters for the amorphous component must be varied during fitting because this phase's composition changes with the progressive crystallization of the sample. As can be seen in this figure, the best fit matches closely the filtered data over the entire $k$-range.

A summary of the fitting results are presented as a function of annealing temperature in Figure 1c. The symbols represent the atomic fraction of each phase present in the annealed samples as determined by fitting the environment around the Fe atoms using EXAFS data 


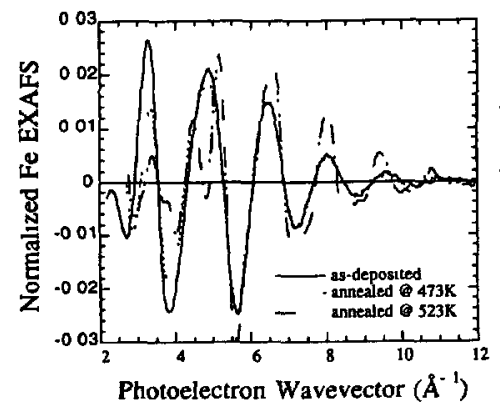

(a)

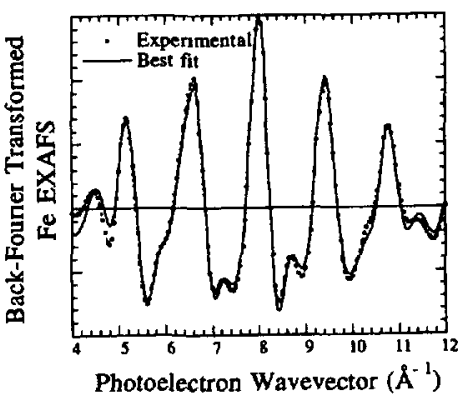

(b)

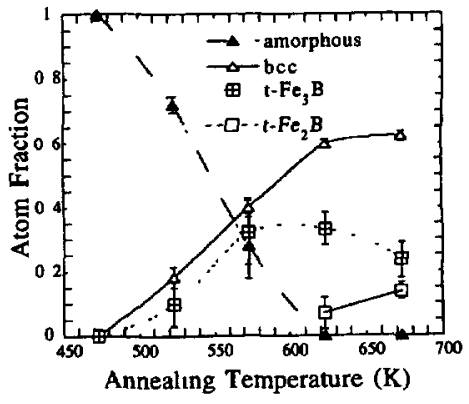

(c)

Fig 1. - (a) EXAFS data for three $\mathrm{Fe}_{80} \mathrm{~B}_{20}$ samples. as-deposited, annealed at $473 \mathrm{~K}$, and annealed at $523 \mathrm{~K}$. Data have been normalized to the absorption edge energy and step height and converted to $k$-space. (b) Experimental, back-Fourier transformed data for the sample annealed at $573 \mathrm{~K}$ together with the best fit obtained using a linear combinations of similarly prepared EXAFS data collected from empirical standards of $\alpha-\mathrm{Fe}, \mathrm{t}-\mathrm{Fe}_{3} \mathrm{~B}$ and amorphous $\mathrm{Fe}_{80} \mathrm{~V}_{20}$. (c) Atomic fraction of amorphous and crystalline components in annealed $\mathrm{Fe}_{80} \mathrm{~B}_{20}$ films determined by EXAFS fitting analysis using empirical standards.

collected from pure phase standards known to be crystallization products of the Fe-B system near this composition. The error bars presented on the symbols of Figure 1c (and in the above text) were determined by systematically varying the fitting parameters until the best fit $\chi^{2}$ parameter doubled in magnitude, signifying the deterioration of the fit. Hence, this "error bar" does not signify an "error" in so much as it provides a means to evaluate the sensitivity of the best fit to the individual parameters used in obtaining the fit. In Figure 1c the initial crystallization is seen to occur with the co-precipitation of $\alpha$-Fe and $t-\mathrm{Fe}_{3} \mathrm{~B}$ : These phases appear together in nearly equivalent fractions. Previous studies by Herold and Koster [8] using thicker samples similarly found that crystallization of the $\mathrm{Fe}_{80} \mathrm{~B}_{20}$ composition occured vaa eutectic crystallization of $\alpha-\mathrm{Fe}$ and $t-\mathrm{Fe}_{3} \mathrm{~B}$. Our results indicate that the eutectic crystallization continues until an annealing temperature of $623 \mathrm{~K}$, at which time the fraction of $\mathrm{t}-\mathrm{Fe}_{3} \mathrm{~B}$ is measured to decrease and the $t-\mathrm{Fe}_{2} \mathrm{~B}$ phase is detected. This behavior is also expected in the crystallization of amorphous $\mathrm{Fe}_{80} \mathrm{~B}_{20}$ where the $\mathrm{t}-\mathrm{Fe}_{2} \mathrm{~B}$ phase forms at the expense of the metastable $\mathrm{t}-\mathrm{Fe}_{3} \mathrm{~B}$ [8] at higher temperature anneals. At still higher temperature anneals the relative amount of the $t-\mathrm{Fe}_{2} \mathrm{~B}$ phase increases at the expense of the $t-\mathrm{Fe}_{3} \mathrm{~B}$ phase. Although the Debye-Waller factors of the crystallized phases in annealed samples may differ from those of the pure phase standards, these differences will be reflected in the uncertainty of the fitting (z.e. error bar) and as such does not limit this approach.

This research was carried out, in part, at the National Synchrotron Light Source (Brookhaven National Laboratories, Upton, NY), which is sponsored by the U.S. Department of Energy.

\section{References}

[1] Swineford R.S., Papas D.P. and Harris V.G., Phys. Rev. B 52 (1995) 7890.

[2] Erbil A., Cargil III G.S., Frahm R. and Boehme R.F., Phys. Rev. B 37 (1988) 2450.

[3] Elam W.T., Kirkland J.P., Neiser R.A. and Wolf P.D., Phys. Rev. B 38 (1988) 26. 
[4] Sayers D.E. and Bunker B.A.. in X-ray Absorption: Principles, Applications, Techniques of EXAFS, SEXAFS and XANES, D.C. Koningsberger and R. Prins, Eds. (New York, Wiley, 1988) p. 211.

[5] Luborsky F.E. and Liebermann H.H., Appl. Phys. Lett. 33 (1978) 233.

[6] Sanchez F.H., Zhang Y.D. and Budnick J.I., Phys. Rev. B 38 (1988) 8508.

[7] Franke H., Herold U., Koster U. and Rosenberg M, Proceedings of the Third International Conference on Rapidly Quenched Metals, vol. 1 (London, Metals Society, 1978).

[8] Herold U. and Koster U., Proceedings of the Third International Conference on Rapidly Quenched Metals, vol. 1 (London, Metals Society, 1978). 